\title{
Lattice-Reduction-Aided Robust Detection and Precoding for MIMO Systems with Imperfect CSI
}

\author{
Jiandong Li, Rui Chen and Wei Liu \\ Information Science Institute \\ State Key Laboratory of Integrated Service Networks, Xidian University \\ Xian, China \\ Email: \{jdli, liuweixd \}@mail.xidian.edu.cn rchenxidian@gmail.com
}

\begin{abstract}
Recently, lattice-reduction (LR) has been proposed to improve the performance of linear and successive interference cancellation (SIC) detectors and precoders in MIMO systems. It is proved that LR-aided linear detectors collect the same diversity order as that exploited by the ML detector but with much lower complexity when perfect channel state information (CSI) is assumed to be available. However, in practice, CSI is always contaminated by channel estimation error and feedback quantization error. In this paper, we proposed MMSE based LRaided robust linear and SIC detectors and MMSE based LRaided (robust) linear precoder and (robust) TomlinsonHarashima precoder (THP) which are capable of improving the BER performance in the context of imperfect CSI. Furthermore, we find that the performance improvement of LR-aided nonlinear method in SIC detectors at receiver is more pronounced than that in THP at transmitter.
\end{abstract}

Keywords-MIMO; lattice reduction; robust design; channel state information; Tomlinson-Harashima precoding.

\section{INTRODUCTION}

Recently, lattice-reduction (LR) has attracted a lot of attention for its potential in improving performance of linear and successive interference cancellation (SIC) detectors and precoders in MIMO systems. LR-aided linear detection was first proposed by Yao and Wornell to transform the system model to an equivalent one with better conditioned channel matrix [1]. In [2], the LR-aided linear and SIC detection schemes have been extended with respect to minimum-meansquare-error (MMSE) criterion. It is proved in [3][4] that LRaided linear detectors collect the same diversity order as that exploited by the maximum-likelihood (ML) detector (i.e., the number of receive antennas) but with much lower complexity. LR-aided zero-forcing (ZF) precoder was put forward in [5] and was shown its superiority in transmit power reduction. It is also illustrated in [6] that their proposed LR-aided TomlinsonHarashima precoding (THP) can achieve the same diversity order as a maximum-likelihood signal generation at the transmitter would offer.

However, the above conclusions are based on the assumption that perfect channel state information (CSI) at receiver (CSIR) or transmitter (CSIT) is available, i.e., there is no channel estimation error and feedback quantization error. The assumption doesn't exist in practical systems. Channel estimation error is inevitable due to the presence of background noise in the estimated signal. In some cases CSIT can be obtained if there is reciprocity between the forward and reverse channels, but more often channel state information needs to be quantized and sent to the transmitter over a limitedrate feedback channel [7]. We can see from the simulation results in [8] and the following section $\mathrm{V}$ that even a very small channel error will lead to pronounced performance degradation for LR-aided detection and precoding. So, the channel error should be considered in practical design. In the present work, we proposed MMSE based LR-aided robust linear detector and precoder, nonlinear SIC detector and THP with the help of complex Lenstra-Lenstra-Lovász (CLLL) algorithm [9], which involve channel estimation error (and feedback quantization error) as an integral part of the LR-aided detector (/precoder) design.

The remainder of the paper is organized as follows. In Section II, we present the system description and the channel error model. In Section III, CLLL algorithm is first introduced, then MMSE based LR-aided robust linear and SIC detection are developed. In Section IV, MMSE based LR-aided (robust) linear precoder and nonlinear THP are brought forward. Simulation results are presented in Section V, and we conclude our work in Section VI.

Notation: Upper (lower) case boldface letters are for matrices (vectors); $(\cdot)^{H}$ denotes complex conjugate transpose (Hermitian) and $(\cdot)^{\dagger}$ stands for Moore-Penrose inverse; $\mathbf{I}_{a}$ is the $a \times a$ identity matrix and $\mathbf{0}$ is the zero-matrix; $\lfloor a\rfloor$ is the largest integer not exceeding $a$ and $\operatorname{diag}(\mathbf{R})$ is a diagonal matrix with elements from the main diagonal of $\mathbf{R}$.

\section{SYSTEM MODEL}

We consider a communication system with $M$ transmit antennas and $N$ receive antennas in a frequency flat fading channel. Let $\mathbf{H}$ be the $N \times M$ channel matrix with independent and identically distributed (i.i.d.) complex Gaussian elements $C N(0,1)$. Let $\mathbf{s} \in \mathbb{C}^{M \times 1}$ represent the transmit signal vector. Let $\mathbf{n}$ denote an complex additive white Gaussian noise vector $C N\left(\mathbf{0}, \sigma_{n}^{2} \mathbf{I}\right)$ and $\mathbf{x} \in \mathbb{C}^{N \times 1}$ denote the received signal, yielding the following relationship with $\mathbf{S}$

$$
\mathbf{x}=\mathbf{H s}+\mathbf{n} .
$$


For open-loop MIMO systems without CSIT, a detector is needed to recover $\mathbf{S}$, then the received signal $\mathbf{x}$ is detected by use of nulling matrix $\mathbf{W}$ at the receiver, yielding the output $\hat{\mathbf{s}}=\mathbf{W x}$. In this case, the relationship between the transmit and detected signals can be written as:

$$
\hat{\mathbf{s}}=\mathbf{W x}=\mathbf{W H s}+\mathbf{W n}
$$

For closed-loop MIMO systems with CSIT, a precoder $\mathbf{F}$ can be used to pre-equalize the effect of $\mathbf{H}$ to $\mathbf{S}$, thus the received signal at receiver can be expressed as

$$
\mathbf{x}=\hat{\mathbf{s}}=\mathbf{H F s}+\mathbf{n}
$$

Due to errors introduced by channel estimation and vector quantization in limited feedback MIMO systems, we assume that the receiver of open-loop MIMO system have only an estimate of the true channel response $\mathbf{H}$ denoted by $\hat{\mathbf{H}}$. The channel estimation error model is

$$
\mathbf{H}=\hat{\mathbf{H}}+\Delta \mathbf{H},
$$

where we assume that the estimation error matrix $\Delta \mathbf{H}$ has $N \times M$ independent elements with zero mean and estimation error variance denoted by $\sigma_{\Delta \mathbf{H}}^{2}$. Moreover, $\Delta \mathbf{H}$ is assumed independent of the data vector $\mathbf{S}$ and the Gaussian noise vector n . The same channel estimation error model was also considered in [10]. Then, we assume the transmitter of closedloop MIMO system have only an quantized estimate of the true channel response $\mathbf{H}$ denoted by $\hat{\mathbf{H}}$. Thus, the channel error is the sum of estimation error and vector quantization error. The channel model is as follows.

$$
\mathbf{H}=\widehat{\mathbf{H}}+\Delta \mathbf{H}+\Delta \breve{\mathbf{H}}=\widehat{\mathbf{H}}+\Delta \mathbf{H}^{\prime \prime},
$$

where the vector quantization error matrix $\Delta \breve{\mathbf{H}}$ has $N \times M$ independent elements with zero mean and error variance denoted by $\sigma_{\Delta \breve{\mathbf{H}}}^{2}$. A similar vector quantization error model was also considered in [11]. Thus, the variance $\sigma_{\Delta \mathbf{H}^{\prime \prime}}^{2}$ of the elements in $\Delta \mathbf{H}^{\prime \prime}$ is equal to $\left(\sigma_{\Delta \mathbf{H}}^{2}+\sigma_{\Delta \breve{H}}^{2}\right)$. Also, $\Delta \mathbf{H}$ is assumed independent of data vector $\mathbf{S}$, Gaussian noise vector $\mathbf{n}$ and estimation error $\Delta \mathbf{H}$.

\section{LR-AIDED RoBUST DETECTION FOR OPEN-LOOP MIMO SYSTEMS}

Given the system model in (1), if the symbols $\mathbf{S}$ are drawn from Gaussian integer ring (e.g., QAM, PAM constellations), then $\mathbf{H s}$ belongs to a lattice spanned by the columns of $\mathbf{H}$ [12]. Hence, to decode $\mathbf{S}$ becomes to find the nearest point to $\mathbf{x}$ on the lattice spanned by $\mathbf{H}$. As we know, when the lattice basis $\mathbf{H}$ is orthogonal, i.e., $\mathbf{H}^{H} \mathbf{H}$ is diagonal, the decision region of linear detectors to find the nearest point is the same as that of ML detector. Therefore, in this case, ZF detector has the same performance as the ML detector. However, in general $\mathbf{H}$ is not orthogonal. Thus, linear equalizers induce performance degradation.
The aim of lattice-reduction is to transform a given basis (columns of $\mathbf{H}$ ) into a new basis (columns of $\tilde{\mathbf{H}}$ ) with vectors of shortest length or, equivalently, into a basis consisting of roughly orthogonal basis vectors. Usually, $\tilde{\mathbf{H}}$ is much better conditioned than $\mathbf{H}$ and therefore leads to less noise enhancement for linear detection.

\section{A. Complex Lenstra-Lenstra-Lovász (CLLL) algorithm}

In theoretical aspect, finding an optimal basis in a lattice is computationally expensive [12]. Two LR methods KorkineZolotareff (KZ) reduction [13] and Lenstra-Lenstra-Lovász (LLL) algorithm [14] are widely used. The KZ algorithm can find the optimal basis for a lattice, but it is highly complex and thus infeasible for practical implementation. The LLL algorithm does not guarantee to find the optimal basis, but it guarantees to find a basis within a factor to the optimal one in polynomial time [14]. A reduced basis for a real lattice is defined in [14], in which the maximum number of arithmetic operations needed by the LLL algorithm to find a new basis is $O\left(K^{4}\right)$, where $K$ is the size of the basis. Most of existing results in [2] [5] adopt the real LLL (RLLL) algorithm in [14] and use the real LR-aided detectors, while [4] and [9] provide a complex LLL (CLLL) algorithm which greatly reduces the RLLL's complexity without sacrificing any performance. Since complexity is a major practical concern, the CLLL algorithm is adopted in this paper.

Following the CLLL algorithm, we find a "better" channel matrix $\tilde{\mathbf{H}}=\mathbf{H T}$ from the original channel matrix $\mathbf{H}$, where $\mathbf{T}$ is a unimodular matrix, which means that all the entries of $\mathbf{T}$ and $\mathbf{T}^{-1}$ are integers and the determinant of $\mathbf{T}$ is \pm 1 or $\pm j$.

\section{B. Robust Linear MMSE Detection Algorithms}

As we know, the MMSE detector takes the noise term into account and thereby leads to an improved performance. As shown in [15], MMSE detection is equal to ZF detection with respect to an extended system model. To this end, the $(N+M) \times M \quad$ extended channel matrix $\underline{\mathbf{H}}$ and the $(N+M) \times 1$ extended receive vector $\mathrm{x}$ is defined as

$$
\underline{\mathbf{H}}=\left[\begin{array}{c}
\mathbf{H} \\
\sqrt{\alpha} \mathbf{I}_{M}
\end{array}\right] \quad \text { and } \quad \underline{\mathbf{x}}=\left[\begin{array}{c}
\mathbf{x} \\
\mathbf{0}_{M, 1}
\end{array}\right] .
$$

where $\alpha=\sigma_{n}^{2} / \sigma_{s}^{2}$. Then, the output of the MMSE detector can be written as

$$
\begin{aligned}
\tilde{\mathbf{s}}_{\text {MMSE }} & =\left(\mathbf{H}^{H} \mathbf{H}+\alpha \mathbf{I}_{M}\right)^{-1} \mathbf{H}^{H} \mathbf{x} \\
& =\left(\underline{\mathbf{H}}^{H} \underline{\mathbf{H}}\right)^{-1} \underline{\mathbf{H}}^{H} \underline{\mathbf{x}}=\underline{\mathbf{H}}^{\dagger} \underline{\mathbf{x}} .
\end{aligned}
$$

Thus, the MMSE detector is equivalent to a ZF detector with respect to the extended system model and consequently the orthogonality of columns of $\underline{\mathbf{H}}$ determines the effective noise amplification. 
When considering the error introduced by channel estimation, we assume that the receiver knows the channel response estimation value $\hat{\mathbf{H}}$ and the statistics of the channel estimation error $\sigma_{\Delta \mathbf{H}}^{2}$. Then, the robust MMSE detector takes the channel estimation error and also noise term into account, leading to an improved performance in the environment with channel estimation error. Similarly, the robust MMSE detection is equal to $\mathrm{ZF}$ detection with respect to a modified $(N+M) \times M$ extended channel matrix $\underline{\hat{\mathbf{H}}}$ [16] expressed as

$$
\underline{\hat{\mathbf{H}}}=\left[\begin{array}{c}
\hat{\mathbf{H}} \\
\sqrt{M \sigma_{\Delta \mathbf{H}}^{2}+\alpha} \mathbf{I}_{M}
\end{array}\right] \text {. }
$$

Then, the output of the robust MMSE detector can be calculated as

$$
\tilde{\mathbf{s}}_{R-M M S E}=\underline{\hat{\mathbf{H}}}^{\dagger} \underline{\mathbf{x}} .
$$

This result will be extremely important for incorporating the robust MMSE method in the following LR-aided detection algorithms.

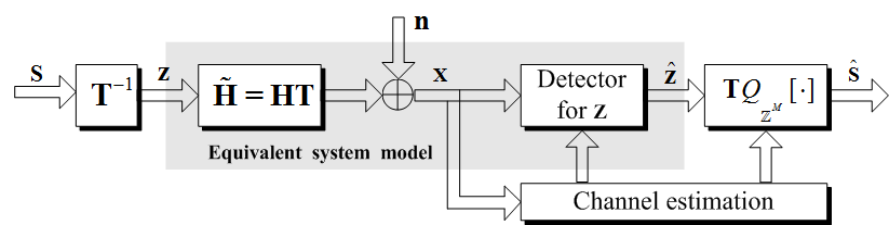

Figure 1. Block diagram of a LR-aided detector for MIMO systems.

\section{LR-Aided Robust Linear MMSE Detection}

As already mentioned, linear detection is optimal for an orthogonal channel matrix. Now, with $\tilde{\hat{\mathbf{H}}}=\hat{\mathbf{H}} \hat{\mathbf{T}}$ and the introduction of $\mathbf{z}=\hat{\mathbf{T}}^{-1} \mathbf{S}$ the receive signal vector (1) can be rewritten as

$$
\begin{aligned}
\mathbf{x} & =\mathbf{H} \mathbf{s}+\mathbf{n}=(\hat{\mathbf{H}}+\Delta \mathbf{H}) \mathbf{s}+\mathbf{n} \\
& =(\hat{\mathbf{H}}+\Delta \mathbf{H}) \hat{\mathbf{T}} \hat{\mathbf{T}}^{-1} \mathbf{s}+\mathbf{n}=\tilde{\hat{\mathbf{H}}} \mathbf{z}+\Delta \tilde{\mathbf{H}} \mathbf{z}+\mathbf{n} .
\end{aligned}
$$

The idea behind LR-aided robust detection is to consider the equivalent system model in (11) and perform the nonlinear quantization on $\mathbf{z}$ instead of $\mathbf{S}$. For LR-aided robust MMSE detection this means that first performing the LR for the extended channel matrix (9), i.e., $\hat{\mathbf{H}}=\hat{\mathbf{H}} \hat{\mathbf{T}}$. Then,

$$
\tilde{\mathbf{z}}_{R-M M S E}=\underline{\hat{\mathbf{H}}}^{\dagger} \underline{\mathbf{x}}=\hat{\mathbf{T}}^{-1} \tilde{\mathbf{S}}_{R-M M S E}
$$

is calculated, where the multiplication with $\underline{\hat{\mathbf{H}}}^{\dagger}$ usually causes less noise amplification and interference amplification caused by channel estimation error than the multiplication with $\hat{\mathbf{H}}^{\dagger}$ in (10) due to the roughly orthogonal columns of $\underline{\hat{\mathbf{H}}}^{\dagger}$. Therefore, a hard decision based on $\tilde{\mathbf{z}}_{R-M M S E}$ is in general more reliable than one on $\tilde{\mathbf{s}}_{R-M M S E}$. Then, the real and imaginary part of $\tilde{\mathbf{z}}_{R-M M S E}$ is independently quantized to the nearest unconstrained integer point in integer space $\mathbb{Z}^{M}$, i.e.,
$\hat{\mathbf{z}}_{R-M M S E}=Q_{{ }_{M}}\left[\tilde{\mathbf{z}}_{R-M M S E}\right] \in \mathbb{Z}^{M}$, where $Q_{{ }^{M}}[\cdot]$ denotes the simple quantization to integer space operation. Finally, the receiver calculates $\hat{\mathbf{s}}_{R-M M S E}=\hat{\mathbf{T}} \hat{\mathbf{z}}_{R-M M S E}$ and restricts $\hat{\mathbf{s}}_{R-M M S E}$ to the original constellation. The block diagram for a LR-aided detector is shown in Fig. 1.

\section{LR-Aided Robust MMSE-SIC Detection}

As $\underline{\hat{\mathbf{H}}}$ is only roughly orthogonal, the mutual interference of the transformed signals $z_{i}$ (the $i$-th element of $\mathbf{z}$ ) is small, but still present. Thus, successive interference cancellation techniques may result in additional improvements. As shown in [15] MMSE-SIC can be well described in terms of the QR decomposition of the expanded channel matrix $\underline{\mathbf{H}}$ in (6). Similar to the previous LR-aided robust MMSE detection, we can develop the LR-aided robust MMSE-SIC detection according to the modified extended channel matrix $\underline{\hat{\mathbf{H}}}$ in (9). Firstly, the $\mathrm{QR}$ decomposition $\underline{\hat{\mathbf{H}}}=\mathbf{Q R}$ is performed. Then,

$$
\tilde{\mathbf{z}}_{R-M M S E-S I C}=\mathbf{Q}^{H} \underline{\mathbf{x}}=\mathbf{R z}+\boldsymbol{\eta}
$$

is computed. Where, the newly defined noise term $\boldsymbol{\eta}$ also incorporates residual interference. Due to the upper triangular structure of $\mathbf{R}$, the $M$-th element $\tilde{z}_{M}$ is free of interference and can be used to estimate $z_{M}$. Proceeding with $\tilde{z}_{M-1}, \ldots, \tilde{z}_{1}$ and assuming correct previous decision in each step, the interference can be perfectly cancelled. After getting $\hat{\mathbf{z}}_{R-M M S E-S I C}, \quad$ Similarly, the receiver calculate $\hat{\mathbf{s}}_{R-M M S E-S I C}^{R-M M S E-S I C}=\hat{\mathbf{T}} \hat{\mathbf{z}}_{R-M M S E-S I C}$ and restrict $\hat{\mathbf{s}}_{R-M M S E-S I C}$ to the original constellation for next-step demodulation.

\section{LR-AIDED ROBUST PRECODING FOR CLOSED-LOOP MIMO SYSTEMS}

The above detection schemes have their corresponding dual forms at the transmitter side, i.e., precoding schemes. Here we propose MMSE based LR-aided precoding and MMSE based LR-aided robust precoding for limited feedback MIMO systems.

\section{A. (Robust) Linear MMSE Precoding}

When $N=M$, the optimal MMSE precoder given perfect CSIT was derived in [17] with output of the precoder written as

$$
\begin{aligned}
\tilde{\mathbf{s}}_{M M S E} & =\mathbf{H}^{H}\left(\mathbf{H H}^{H}+\frac{N}{\rho} \mathbf{I}_{N}\right)^{-1} \mathbf{s} \\
= & \mathbf{A} \underline{\underline{H}}^{H}\left(\underline{\underline{\mathbf{H}}}={ }^{H}\right)^{-1} \mathbf{s}=\mathbf{A} \underline{\underline{H}}^{\dagger} \mathbf{s},
\end{aligned}
$$

where $\rho$ is the ratio of total transmit power to noise power. $N \times(M+N)$ extended channel matrix $\underline{\mathbf{H}}$ and the $M \times(M+N)$ matrix $\mathbf{A}$ is defined as

$$
\underline{\underline{\mathbf{H}}}=\left[\mathbf{H}, \sqrt{\frac{N}{\rho}} \mathbf{I}_{N}\right] \quad \text { and } \quad \mathbf{A}=\left[\mathbf{I}_{M}, \mathbf{0}_{M \times N}\right] .
$$


Note that $\mathbf{A} \mathbf{A}^{H}=\mathbf{I}_{M}$, so the term $\mathbf{A}$ in precoding matrix $\mathbf{A H}^{\dagger}$ doesn't result in transmit power amplification. When considering the error introduced by channel estimation and vector quantization, we assume that the transmitter knows the quantized estimation value $\hat{\mathbf{H}}$, error variance $\sigma_{\Delta \mathbf{H}}^{2}$ and $\sigma_{\Delta \breve{\mathbf{H}}}^{2}$. Then, the robust MMSE precoder [11] takes the channel error and noise term into account, leading to an improved performance in the environment with imperfect CSIT. The robust MMSE precoding is equal to $\mathrm{ZF}$ precoding with respect to a modified $(N+M) \times M$ extended channel matrix $\underline{\hat{\mathbf{H}}}$ expressed as

$$
\underline{\hat{\mathbf{H}}}=\left[\hat{\mathbf{H}}, \sqrt{N \cdot\left(\left(\sigma_{\Delta \mathbf{H}}^{2}+\sigma_{\Delta \breve{\mathbf{H}}}^{2}\right)+\frac{1}{\rho}\right)} \mathbf{I}_{N}\right] .
$$

Thus, the output of the robust MMSE precoder can be calculated as

$$
\tilde{\mathbf{s}}_{R-M M S E}=\mathbf{A} \underline{\underline{\hat{\mathbf{H}}}}^{\dagger} \mathbf{s} .
$$

The above results will be used to incorporat MMSE method in the following LR-aided (robust) precoding algorithms.

\section{B. LR-Aided (Robust) Linear MMSE Precoding}

For traditional ZF based LR-aided precoding [5][6], LR is performed on $\mathbf{H}^{T}$. Instead of performing CLLL reduction on $\mathbf{H}^{H}$, we perform CLLL reduction on $\underline{\mathbf{H}}^{H}$ and $\underline{\hat{\mathbf{H}}}^{H}$ respectively for $\mathrm{LR}$-aided precoding and $\mathrm{LR}$-aided robust precoding, because the orthogonality of rows of $\underline{\underline{H}}$ and $\underline{\hat{\mathbf{H}}}$ determine the effective transmit power amplification.

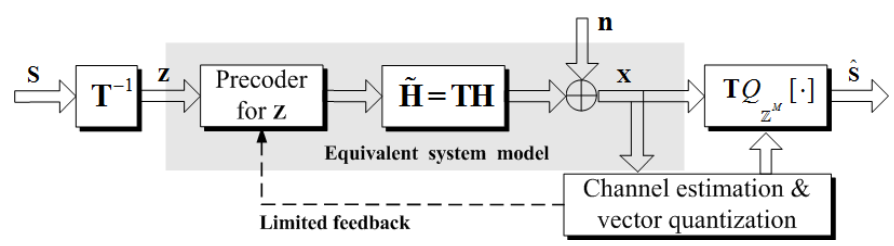

Figure 2. Block diagram of a LR-aided precoder for limited feedback MIMO systems.

For LR-aided precoding given perfect CSIT, with $\tilde{\tilde{\mathbf{H}}}=\mathbf{T H}$ by preprocessing the transmit signal with $\underline{\underline{\mathbf{H}}}^{\dagger}$, we obtain the receive signal

$$
\mathbf{x}=\mathbf{H A} \underline{\underline{\tilde{H}}}^{\dagger} \mathbf{s}+\mathbf{n},
$$

and the estimate for $\mathbf{s}$ is given by

$$
\hat{\mathbf{s}}=\mathbf{T} Q_{\mathbb{Z}^{M}}[\mathbf{x}] .
$$

The property of $\underline{\tilde{\mathbf{H}}}$ consisting of close-to-orthogonal rows leads to a significant reduction in required transmit power of preequalized transmit signal $\tilde{\mathbf{H}}^{\dagger} \mathbf{s}$ compared with that of linear preequalization via $\underline{\underline{\mathbf{H}}}^{\dagger}$ in $(15 \overline{\overline{)}}$.

For LR-aided robust precoding in limited feedback MIMO systems, with $\hat{\hat{\mathbf{H}}}=\hat{\mathbf{T}} \hat{\underline{\mathbf{H}}}$ by preprocessing the transmit signal with $\underline{\underline{\mathbf{H}}}^{\dagger}$, we obtain the receive signal

$$
\mathbf{x}=\mathbf{H A} \underline{\underline{\hat{\mathbf{H}}}}^{\dagger} \mathbf{s}+\mathbf{n}
$$

and the estimate for $\mathbf{s}$ is given by

$$
\hat{\mathbf{s}}=\hat{\mathbf{T}} Q_{\mathbb{Z}^{M}}[\mathbf{x}] .
$$

The block diagram for a LR-aided precoder in a limited feedback MIMO system is shown in Fig. 2.

\section{LR-Aided (Robust) Tomlinson-Harashima Precoding}

As aforementioned, SIC detector can further improve the performance of linear detector, so THP may also be combined with LR to enhance the performance of LR-aided linear precoder. LR-aided ZF THP was proposed in [6]. Here we propose MMSE based LR-aided THP and MMSE based LRaided robust THP.

It is claimed that MMSE based THP first proposed in [18] can be implemented through QR decomposition of the following product of matrices expressed as

$$
\mathbf{H}^{-1}\left(\mathbf{H} \mathbf{H}^{H}+\frac{N}{\rho} \mathbf{I}\right)=\mathbf{Q R} \text {. }
$$

However, we perform QR decomposition on $\underline{\tilde{\mathbf{H}}}^{H}$ for MMSE based LR-aided THP $\underset{\overline{\mathbf{H}}}{\tilde{T}}=\mathbf{R}^{H} \mathbf{Q}^{H}$. Then, using successive interference precancellation and modulo operation of THP, the output of the precoder is

$$
\tilde{\mathbf{s}}_{M M S E-T H P}=\mathbf{A} \mathbf{Q} \cdot \Gamma_{L}\left[\mathbf{R}^{-H} \operatorname{diag}(\mathbf{R}) \mathbf{T s}\right] .
$$

where modula arithmetic operation $\Gamma_{L}[\bullet]$ is used to bound the value of the transmitted signal [6][20] layer by layer and $L$ is the size of modulation. At receiver, we obtain the estimate of $\mathbf{s}$ given by

$$
\hat{\mathbf{s}}_{\text {MMSE-THP }}=Q_{\mathbb{Z}^{M}}\left[\Gamma_{L}\left[\operatorname{diag}(\mathbf{R})^{-1} \mathbf{x}\right]\right] .
$$

Similarly, QR decomposition is performed on $\tilde{\hat{\mathbf{H}}}^{\dagger H}$ for MMSE based robust LR-aided THP expressed as $\underline{\hat{\mathbf{H}}}=\overline{\overline{\mathbf{R}}}{ }^{H} \mathbf{Q}^{H}$. Then, using successive interference precancellation and modulo operation, the output of the precoder is

$$
\tilde{\mathbf{s}}_{R-M M S E-T H P}=\mathbf{A Q} \cdot \Gamma_{L}\left[\mathbf{R}^{-H} \operatorname{diag}(\mathbf{R}) \hat{\mathbf{T}} \mathbf{s}\right] .
$$

At receiver, the estimate of $\mathbf{s}$ is obtained by

$$
\hat{\mathbf{s}}_{R-M M S E-T H P}=Q_{\mathbb{Z}^{M}}\left[\Gamma_{L}\left[\operatorname{diag}(\mathbf{R})^{-1} \mathbf{x}\right]\right] .
$$

\section{Simulation Results}

In this section, computer simulation results are presented to evaluate the performance of the MMSE based LR-aided robust detection and (robust) precoding schemes proposed in this paper. In the simulation, we use the channel model presented in Section II. The SNR is defined as symbol energy per transmit antenna versus noise power. 
The simulation results are based on $10^{6}$ Monte Carlo realizations of $\widehat{\mathbf{H}}, \Delta \mathbf{H}$ and $\Delta \breve{\mathbf{H}}$. In Figure 3, we assume a QPSK modulation and plot the average BER of the proposed robust LR-aided MMSE detection as a function of the SNR at three different channel estimation errors. For comparison, on the same figure we plot the performance of the traditional LRaided MMSE detection. As we can see from the figure, for relative high SNR, the proposed robust LR-aided MMSE detection outperforms the traditional LR-aided MMSE detection, and the improvement increases with the SNR in a certain SNR range. We can see the ceiling effect that the average BER of both detection schemes flattens at high SNR and does not improve by increasing the SNR. However, using the proposed robust detection the error floor has been pulled down by, for $\sigma_{\Delta \mathbf{H}}=0.15$ about 30 percent, for $\sigma_{\Delta \mathbf{H}}=0.1$ about 43 percent and for $\sigma_{\Delta \mathbf{H}}=0.05$ about 73 percent.

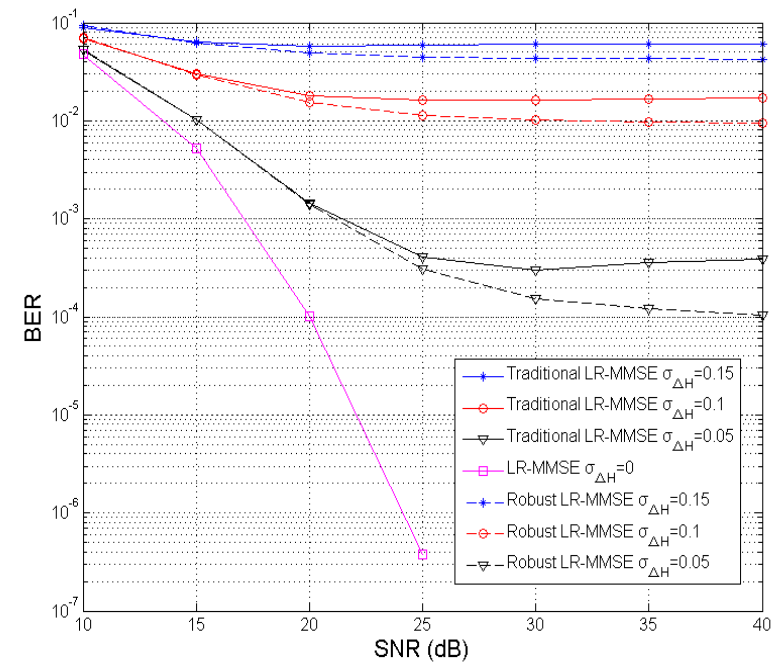

Figure 3. BER comparison for traditional LR-aided MMSE detection and robust LR-aided MMSE detection using QPSK with $N=4, M=4$.

In Figure 4, we observe that both the traditional and the robust LR-aided MMSE-SIC detection have a better performance than pure MMSE detection without SIC. Because SIC further remove the mutual interference of the transformed signals $z_{i}$. For relative high SNR, the proposed robust LRaided MMSE-SIC detection outperforms the traditional LRaided MMSE-SIC detection when there exists channel estimation error. For LR-aided MMSE-SIC detection, the proposed robust scheme has pulled down the ceiling by, for $\sigma_{\Delta \mathbf{H}}=0.15$ about 35 percent, for $\sigma_{\Delta \mathbf{H}}=0.1$ about 52 percent and for $\sigma_{\Delta \mathbf{H}}=0.05$ about 80 percent.

In Figure 5, the BER performance of traditional MMSE based linear precoding, MMSE based LR-aided linear precoding and MMSE based LR-aided robust linear precoding is compared. We can see the curve of MMSE based linear precoding and MMSE based LR-aided robust linear precoding coincides when $\sigma_{\Delta \mathbf{H}^{\prime \prime}}=0$, which is evident from the equations in section III. Moreover, the MMSE based LR-aided robust

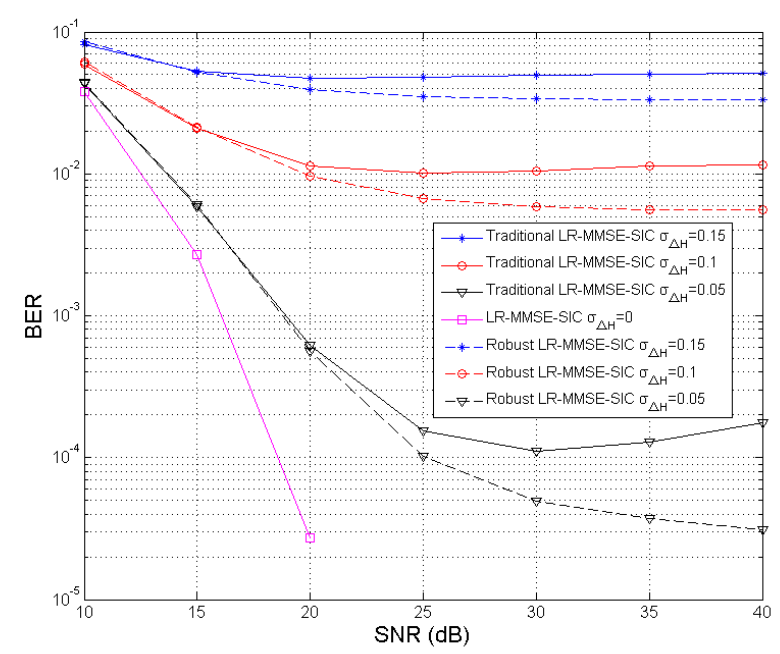

Figure 4. BER comparison for traditional LR-aided MMSE-SIC detection and robust LR-aided MMSE-SIC detection using QPSK with $N=4, M=4$.

linear precoding outperforms MMSE based LR-aided linear precoding in a relative high SNR region. The error floor has been pulled down by, for $\sigma_{\Delta \mathbf{H}}=0.05$ about 70 percent. Note that both LR-aided precoding schemes have some performance loss in low SNR region compared with traditional MMSE based linear precoding, because when noise power is relatively high, there exists some performance loss in the process of decision on a parallelogram lattice rigion.

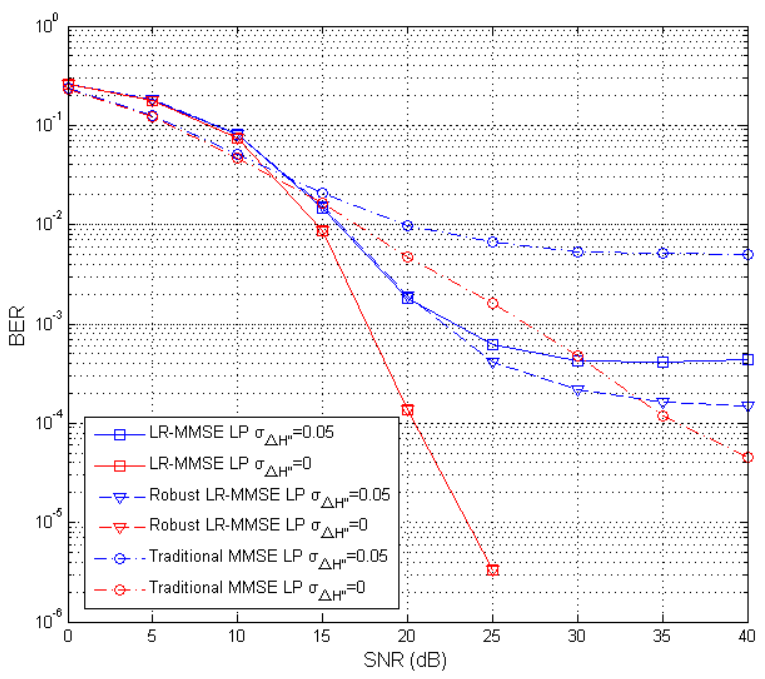

Figure 5. BER comparison for LR-aided MMSE based linear precoding and LR-aided MMSE based robust linear precoding using QPSK with

$$
N=4, M=4 \text {. }
$$

In Figure 6, we plot the BER curves of MMSE based LRaided THP and MMSE based LR-aided robust THP. Comparing Figure 5 with Figure 6, It can be seen that when $\sigma_{\Delta \mathbf{H}^{\prime \prime}}=0$, the curve of MMSE based LR-aided robust THP is lower than MMSE based linear precoding at SNRs higher than $20 \mathrm{~dB}$, while when $\sigma_{\Delta \mathbf{H}^{\prime \prime}}=0.05$, the curve of MMSE based 
LR-aided (robust) THP is almost the same as that of MMSE based LR-aided (robust) linear precoding. The performance improvement of the LR-aided nonlinear sequential interference cancellation in detection at receiver is more pronounced than that in precoding at transmitter.

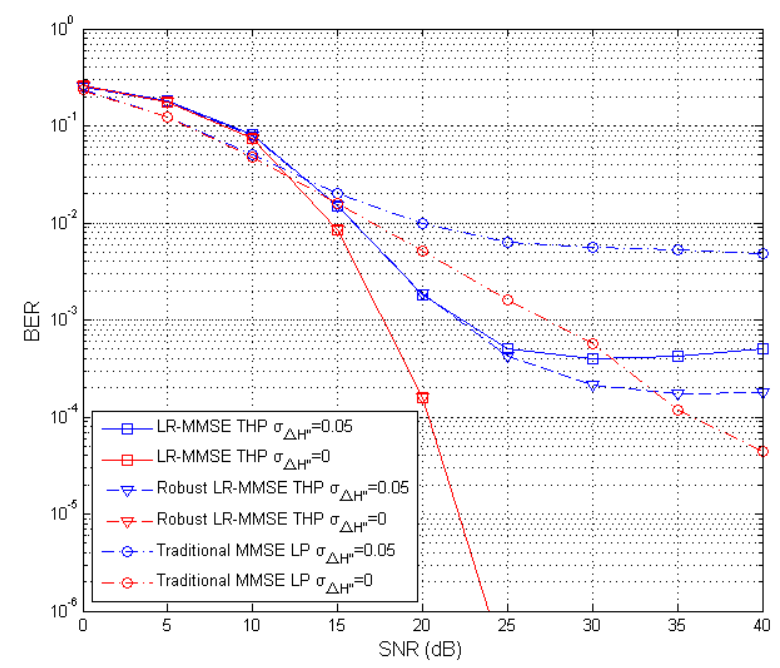

Figure 6. BER comparison for LR-aided MMSE based THP and LR-aided MMSE based robust THP using QPSK with $N=4, M=4$.

\section{CONCLUSIONS}

In this paper, we proposed MMSE based LR-aided robust linear and SIC detection schemes, and MMSE based LR-aided (robust) linear precoding and (robust) THP with the help of complex Lenstra-Lenstra-Lovász (CLLL) algorithm. LR is applied to an extended channel matrix, with which we consider channel error as an integral part of the LR-aided detector and precoder design. Simulation results show that for relative high SNR, the proposed robust LR-aided MMSE based linear and nolinear algorithms outperform the non-robust counterparts respectively, pulling down the error floors to some extent. Furthermore, we observe that the performance improvement of LR-aided nonlinear method in SIC detectors at receiver is more pronounced than that in THP at transmitter.

\section{ACKNOWLEDGMENT}

This work was supported by the National Science Fund for Distinguished Young Scholars (60725105), the National Basic Research Program of China (2009CB320404), the Program for Changjiang Scholars and Innovative Research Team in University, the National High Technology Research and Development Program of China (2007AA01Z288), the 111 Project (B08038), the National Nature Science Foundation of China (60702057), the Key Project of Chinese Ministry of Education (107103), the Fundamental Research Funds for the Central Universities (JY10000901030) and ISN02080001.

\section{REFERENCES}

[1] H. Yao and G. Wornell, .Lattice-Reduction-Aided Detectors for MIMO Communication Systems,. in Proc. IEEE Global Communications Conference, Taipei, Taiwan, November 17-21 2002.

[2] D. Wübben, R. Böhnke, V. Kühn, and K.-D. Kammeyer, "Nearmaximum-likelihood detection of MIMO systems using MMSEbased lattice reduction," in Proc. IEEE International Conference on Communications, vol. 2, pp. 798-802, Paris, France, Jun. 20-24, 2004.

[3] M. Taherzadeh, A. Mobasher and A. K. Khandani, "LLL Reduction Achieves the Receiver Diversity in MIMO Decoding," IEEE Trans. Inf. Theory, vol. 53, no. 12, pp. 4801-4805, Dec. 2007.

[4] X. Ma and W. Zhang, "Performance Analysis for MIMO Systems with Lattice-Reduction Aided Linear Equalization," IEEE Trans. Commun., vol. 56, no. 2, pp. 309-318, Feb. 2008.

[5] C. Windpassinger and R. F. H. Fischer, "Low-complexity near maximum-likelihood detection and precoding for MIMO systems using lattice reduction," in Proc. Information Theory Workshop, pp. 345-348, Munich, Germany, Mar. 31-Apr. 4, 2003.

[6] C. Stierstorfer and R.F.H. Fischer, "Lattice-reduction-aided TomlinsonHarashima precoding for point-to-multipoint transmission", Int. J. Electron. Commun. (AË̈) 60 (2006) 328 - 330.

[7] D. J. Love, R. W. Heath, Jr., W. Santipach, and M. L. Honig, "What is the value of limited feedback for MIMO channels?" IEEE Comm. Mag., vol. 42, no. 10, pp. 54-59, Oct 2004.

[8] C. Windpassinger. Detection and Precoding for Multiple Input Multiple Output Channels. PhD thesis, Universität Erlangen-Nürnberg, Germany, 2004.

[9] Y. H. Gan and W. H. Mow, "Complex lattice reduction algorithms for low-complexity MIMO detection," in Proc. IEEE Global Telecommunications Conf., vol. 5, pp. 2953-2957, St. Louis, USA, Nov. 28-Dec. 2, 2005.

[10] T. Yoo and A. J. Goldsmith, "Capacity and power allocation for fading MIMO channels with channel estimation error," IEEE Trans. Inf. Theory, vol. 52, no. 5, pp. 2203-2214, May 2006.

[11] A. D. Dabbagh and D. J. Love, "Multiple Antenna MMSE Based Downlink Precoding with Quantized Feedback or Channel Mismatch," IEEE Trans.Wireless Commun., vol. 56, no. 11, pp. 1859-1868, Nov. 2008.

[12] E. Agrell, T. Eriksson, A. Vardy, and K. Zeger, "Closest point search in lattices," IEEE Trans. Inf. Theory, vol. 48, no. 8, pp. 2201-2214, Aug. 2002.

[13] C. P. Schnorr and M. Euchner, "Lattice basis reduction: improved practical algorithms and solving subset sum problems," Math. Programming, vol. 66, pp. 181-191, 1994.

[14] A. K. Lenstra, H. W. Lenstra, and L. Lovász, "Factoring polynomials with rational coefficients," Math. Ann, vol. 261, pp. 515-534, 1982.

[15] B. Hassibi, "A fast square-root implementation for BLAST," in Proc. Thirty-Fourth Asilomar Conf. Signals, Syst., Comput., Nov. 2000, pp. 1255-1259.

[16] R. Chen, J. D. Li and W. Liu, "Robust Uniform Channel Decomposition for MIMO Communications," in Proc. IEEE Personal, Indoor and Mobile Radio Communications Symposium, Tokyo, Japan, Sep. 13-16, 2009.

[17] C. B. Peel, B. M. Hochwald, and A. L. Swindlehurst, "A vector perturbation technique for near capacity multiantenna multiuser communication - part I: channel inversion and regularization," IEEE Trans. Commun., vol. 53, no. 1, pp. 195-202, Jan. 2005.

[18] J. Liu and W. A. Krzymien, "Improved Tomlinson-Harashima Precoding for the Downlink of Multiple Antenna Multi-User Systems," in Proc. IEEE Wireless Communications and Networking Conf., vol. 1, pp. 466-472, New Orleans, USA, Mar. 13-17, 2005.

[19] H. K. Bizaki and A. Falahati, "Tomlinson-Harashima precoding with imperfect channel state information," IET Commun., vol. 2, no. 1, pp. 151-158, 2008.

[20] A. A. D'Amico, “Tomlinson-Harashima Precoding in MIMO Systems: A Unified Approach to Transceiver Optimization Based on Multiplicative Schur-Convexity," IEEE Trans. Signal Process., vol. 56, no. 8, pp. 3662-3677, Aug. 2008. 